\title{
Plasma omega-3 PUFA and white matter mediated executive decline in older adults
}

\section{Gene L. Bowman ${ }^{*}$, Hiroko H. Dodge ${ }^{1}$, Nora Mattek ${ }^{1}$, Aron K. Barbey ${ }^{2}$, Lisa C. Silbert ${ }^{1}$, Lynne Shinto ${ }^{1}$, Diane B. Howieson ${ }^{1}$, Jeffrey A. Kaye ${ }^{1}$ and Joseph F. Quinn ${ }^{1}$}

1 Brain Institute, Department of Neurology, Oregon Health and Science University, Portland, OR, USA

${ }^{2}$ Beckman Institute for Advanced Science and Technology, Urbana, IL, USA

\section{Edited by:}

P. Hemachandra Reddy, Oregon

Health and Science University, USA

\section{Reviewed by:}

P. Hemachandra Reddy, Oregon

Health and Science University, USA

Peizhong Mao, Oregon Health and

Science University, USA

\section{*Correspondence:}

Gene L. Bowman, Brain Nutrition Laboratory, Aging and Alzheimer's

Disease Center, Oregon Health and Science University, Mail code:

CR-131, 3181 SW Samuel Jackson

Park Road, Portland, OR 97239, USA

e-mail: bowmang@ohsu.edu
Introduction: Cross-sectional studies have identified long chain omega-3 polyunsaturated fatty acids (eicosapentaenoic acid 20:5n-3 and docosahexaenoic acid 22:6n-3 (O3PUFA) in association with fewer white matter lesions and better executive function in older adults. We hypothesized that O3PUFA are associated with less executive decline over time and that total white matter hyperintensity volume $(\mathrm{WMH})$ mediates this association.

Methods: Eighty-six non-demented older adults were followed over 4 years after measurement of plasma O3PUFA with annual evaluations of cognitive function. A subset of these participants also had brain MRI of total WMH available to conduct a formal mediation analysis of a putative relationship between O3PUFA and cognitive function.

Results: Mean age at baseline was $86,62 \%$ were female and $11 \%$ carried the APOE4 allele. Each $100 \mu \mathrm{g} / \mathrm{ml}$ increase in plasma O3PUFA associated with $4 \mathrm{~s}$ less change in executive decline per year of aging ( $p=0.02$, fully adjusted model). O3PUFA was not associated with verbal memory or global cognitive changes. The significance of the association between O3PUFA and better executive function was lost once WMH was added to the regression model.

Conclusion: Executive decline with age appears to be a cognitive domain particularly sensitive to plasma O3PUFA in longitudinal examination. O3PUFA may modulate executive functioning by mechanisms underlying the development of $\mathrm{WMH}$, a biologically plausible hypothesis that warrants further investigation.

Keywords: omega-3 fatty acids, white matter hyperintensity, cognitive decline, memory, hypertension

\section{INTRODUCTION}

Delaying the onset of Alzheimer's disease and other late life dementias is an agenda budding across the globe. Characterizing the heterogeneity in structural and functional brain changes that relate to dementia risk will enable preventive therapies to target these characteristics in populations primed to gain benefit. Diet and nutrition pose a significant opportunity for prevention; however, success here also requires knowledge of the risk profile in order to engage appropriately.

Cerebral white matter hyperintensities (WMH), largely considered a marker of small vessel disease and dementia risk, are of significant interest to prevention strategies (Schmidt et al., 2003; Garde et al., 2005; Bastos Leite et al., 2006; Kramer et al., 2007; Silbert et al., 2008; Debette et al., 2010; Gorelick et al., 2011). These WMH are prevalent in $60-92 \%$ of non-demented elders age 65 and older and increase risk for cognitive impairment (de Leeuw et al., 2001; Silbert et al., 2009). WMH represents an early structural MRI risk factor for Mild Cognitive Impairment and dementia (Silbert et al., 2012), and thus, provides an attractive therapeutic target. The design of rational therapy for this early brain structural change requires knowledge of its pathophysiology. Both intake (Virtanen et al., 2008) and peripheral concentration of long chain omega-3 polyunsaturated fatty acids
(O3PUFA) are associated with less WMH in cross-sectional studies (Bowman et al., 2012; Tan et al., 2012; Virtanen et al., 2013). Although the cognitive consequences associated with WMH accumulation may ultimately pervade several domains, executive dysfunction with impaired information processing and cognitive flexibility have been noted as early indicators of its accrual (Schmidt et al., 1993; DeCarli et al., 1995; Adak et al., 2004; Brickman et al., 2006; Verdelho et al., 2007). Intrigued by this fabric of literature, we tested the hypothesis that O3PUFA associates with less executive decline, and that WMH mediates the relationship between these fatty acids and executive function.

\section{MATERIALS AND METHODS STUDY POPULATION}

The Oregon Brain Aging Study (OBAS) is a cohort study of brain aging in people age 65 and older free of usual confounding factors known at the time to modify the risk for cognitive decline (i.e., vascular disease, smoking, stroke, diabetes) (Kaye et al., 1994). Enrollment was opened in 2004 to also include volunteers with stable chronic conditions common with advanced age (i.e., hypertension, diabetes) to better represent the general population. Clinical, neuropsychometric and brain MRI are collected annually. Clinical Dementia Rating (CDR) (Morris, 1993) scale is 
based on interviews with the participant and their collateral historian about functioning and cognitive skills in conjunction with the MMSE (Folstein et al., 1975) and the Cognistat (Kiernan et al., 1987). Blood was collected appropriately for nutrient biomarkers beginning in the 2006-2007. Inclusion was restricted to nondemented participants $(C D R \leq 0.5)$. Eighty-six participants had plasma fatty acids and psychometric measures available for longitudinal analysis. Thirty-two of these 86 also had MRI collected at the time of the nutrient blood draw to permit a formal mediation analysis.

\section{STANDARD PROTOCOL APPROVAL AND PATIENT CONSENT}

Informed consent was obtained from all subjects for participation in this study, which was approved by the institutional review board for human study at Oregon Health \& Science University.

\section{BIOMARKER ACQUISITION AND ANALYSIS Plasma long-chain omega-3 fatty acids}

Fasting blood was collected between the hours of 0700 and 1200 noon Pacific Standard Time beginning in September of 2006 and ending December 2007. Total lipid long chain n-3 polyunsaturated fatty acids as methyl esters were quantified using gas chromatography equipped with flame ionization detector and expressed as absolute plasma concentrations $(\mu \mathrm{g} / \mathrm{mL})$ (Bowman et al., 2012). Plasma eicosapentaenoic acid (20:5n-3) and docosahexaenoic acid (22:6n-3) were combined (O3PUFA).

\section{Volumetric brain MRI}

Brain regions of interest were obtained using MRI 1.5 T magnet and REGION image analysis software. The procedures have been previously described (Mueller et al., 1998). Briefly, the sums of pixel areas for all slices were converted to volumetric measures by multiplying by the slice thickness for each of the following regions of interest: total white matter hyperintensity volume (WMH includes periventricular and subcortical deep signals) and supratentorial brain volume as total cerebral brain volume (TBV, excluding cerebellum and brain stem). Regression for brain tissue, CSF, and WMH collectively against bone creates a boundary along the inner table of the skull to determine the total intracranial volume. Additional boundaries were manually traced along the tentorium cerebelli and the superior border of the superior colliculus, the pons, and the fourth ventricle. The pituitary, vessels in the sphenoid region, and any sinuses that may have been included by the automatic regression were excluded manually. Using REGION's sampling tools, the analyst selects representative, unambiguous pixels of WMH (as well as brain tissue, fluid, and bone) from the multi-echo sequence display. Proton and T2 intensities are included in a regression model taking into account the location of each pixel that differentiates the tissue types. Distinction of WMH from brain tissue and fluid is achieved by visualizing higher signal intensities on proton density and T2 images. Inter-rater reliability coefficients using this approach for white matter segmentation are 0.85 and $>0.95$ for total intracranial, brain, and ventricular regions of interest.

\section{PRIMARY OUTCOMES AND POTENTIAL CONFOUNDERS Neuropsychometrics}

Previous cross-sectional analysis (Bowman et al., 2012) demonstrated O3PUFA in association with Trail Making Test Part B (Reitan, 1958), a measure commonly utilized to reflect executive function. This test was therefore used as our primary outcome measure for the current longitudinal analysis. WMS-R Logical Memory Story delayed (Wechsler, 1981) and the MMSE were also analyzed to resolve cognitive measures with apparent sensitivity to O3PUFA over time.

\section{Potential confounders and other covariates}

We utilized a parsimonious approach to our model building by including potential confounders on the basis of their previous association with cognitive decline. These included age (continuous), gender (man/women), education (continuous, years), APOE genotype determined using PCR (e4 carrier, y/n), hypertension $(y / n)$, and depression $(y / n)$. We restricted our covariates entered into the mediation analysis on the basis of their significant association with our outcomes of interest to avoid depleting degrees of freedom in a limited sample size (Supplementary Material, age and APOE4 carrier status, total intracranial volume). Covariates were collected and confirmed during the clinical interview (i.e., hypertension, depression).

\section{STATISTICAL ANALYSIS}

All statistics were performed in STATA v10.1 software (College Station, TX). Baseline differences in characteristics between those with and without MRI were calculated using independent t-test or Wilcoxon rank sum test for continuous variables and Pearson's chi-square test or Fisher's exact test for categorical variables as appropriate.

\section{Longitudinal analysis}

Linear mixed effects models estimated the mean and withinperson slope of cognitive change by baseline plasma O3PUFA concentration. The mixed effects model accounts for the withinperson correlations on repeated measures. The interaction term (O3PUFA x Age) represents the "effects" of the baseline O3PUFA on cognitive change over time (using age at visit as the time variable). We interpret this as the annual cognitive change per unit increase in baseline O3PUFA. In addition to considering O3PUFA as a continuous measure, we also examined the difference between O3PUFA 1-SD above the mean $(>100 \mu \mathrm{g} / \mathrm{ml})$ versus a lesser value in relation to cognitive change. O3PUFA dichotomized ( $>100 \mu \mathrm{g} / \mathrm{ml}$ or less) in this setting represents the between-group annual difference in slope of cognitive change (high vs. low).

\section{Formal mediation analysis}

This procedure was utilized in an attempt to better describe the role of O3PUFA in relation to $\mathrm{WMH}$ and cognitive function. Differences between participants with and without MRI are presented in Table 1. Our mediation analysis used the baseline data and a 3-step framework (Baron and Kenny, 1986). The first step attempts to reproduce the initial model 
Table 1 | Baseline characteristics of the study popualtion ${ }^{a}$.

\begin{tabular}{|c|c|c|c|c|}
\hline & $n=86$ & \multicolumn{2}{|c|}{ MRI available } & $P$ for difference ${ }^{b}$ \\
\hline Female, $n(\%)$ & $53(62)$ & $28(52)$ & $25(78)$ & 0.02 \\
\hline Years of education & $15(3)$ & $15.3(2.6)$ & $14.5(2.4)$ & 0.12 \\
\hline APOE4 carrier, $n(\%)$ & $9(11)$ & $8(15)$ & $1(3)$ & 0.14 \\
\hline CDR of $0, n(\%)$ & $60(70)$ & $38(70)$ & $22(69)$ & 0.87 \\
\hline \multicolumn{5}{|l|}{ PLASMA NUTRIENTS } \\
\hline EPA $(20: 5 n-3), \mu \mathrm{g} / \mathrm{ml}$ & $16.5(10.5)$ & $16.9(12.4)$ & $15.8(7.8)$ & 0.66 \\
\hline DHA (22:6n-3), $\mu \mathrm{g} / \mathrm{ml}$ & $68.1(17.8)$ & $67.1(17.9)$ & $69.6(17.6)$ & 0.48 \\
\hline O3PUFA (EPA+DHA) & $84.5(26.7)$ & $84.1(28.1)$ & $85.4(24.6)$ & 0.61 \\
\hline Trails B & $137(77)$ & $123(73)$ & $160(79)$ & 0.01 \\
\hline Paragraph recall & $14.0(4.7)$ & $15.0(3.8)$ & $12.3(5.6)$ & 0.02 \\
\hline \multicolumn{5}{|l|}{ VOLUMETRIC MRI, cc } \\
\hline Intracranial & - & - & $1107.5(106.4)$ & - \\
\hline Brain & - & - & $821.1(84.7)$ & - \\
\hline Ventricular & - & - & $55.2(16.5)$ & - \\
\hline WMH & - & - & $13.8(8.8)$ & - \\
\hline
\end{tabular}

${ }^{a}$ Mean and standard deviation unless otherwise stated.

${ }^{b}$ Baseline differences between those with and without MRI calculated by independent t-test or Wilcoxon rank sum test for continuous variables and Pearson's chi-square or Fisher's exact test for categorical variables as appropriate.

APOE4, Apolipoprotein E epsilon 4 allele carrier; EPA, eicosapentaenoic acid; DHA, docosahexaenoic acid; Trails B, Trail Making Test - Part B; Paragraph recall, Wechsler memory scale revised; WMH, total white matter hyperintensity.

that demonstrates the association between O3PUFA and cognitive function. In the second step, we examine the association between the proposed "mediator" (i.e., WMH) and cognitive function and O3PUFA with the mediator WMH. The third step includes O3PUFA and WMH as simultaneous predictors of cognitive function. Attenuation of the beta-coefficient $>10 \%$ or loss of statistical significance (alpha level $>0.05$ ) was stated a priori to imply a mediation effect in this construct. These regression models were adjusted for variables that demonstrated a significant association with cognitive function in the study sample (alpha value $<0.05$, two-sided) (Supplementary Material).

\section{RESULTS}

Sixty-two percent of the participants were female and the mean age at baseline was 86 . Prevalence of APOE4 allele carrier status was $11 \%$ (Table 1). The mean MMSE was 28. Forty-two percent were being treated for hypertension and $17 \%$ for depression. Vitamin B12 deficiency was prevalent in 4\%. Mean duration of follow-up was 3.9 years (range 1-5) (Table 1). Annual change in Trail Making Test Part B (Trails B) as measured by speed in test performance was $3.5( \pm 0.42) \mathrm{s},-0.2( \pm 0.04)$ points on WMS-R Delayed Paragraph Recall, and $-0.1( \pm 0.02)$ points on MMSE.

In the results that follow, all subjects are included in the evaluation of relationships between O3PUFA and cognitive change
( $n=86$ ), while only those with MRI are included in the mediation analysis $(n=32)$. The participants with MRI were older and predominantly female in comparison to those without MRI (Table 1).

\section{PLASMA 03PUFA AND COGNITIVE DECLINE (TABLE 2)}

Plasma O3PUFA (EPA+DHA) was associated with less executive decline (Trails B) after adjustment for age, gender, education, APOE4, hypertension, and depression $(p=0.02)$ (Table 2). The magnitude of the association can be interpreted as a $4 \mathrm{~s}$ less decline on Trails B per year for each $100 \mu \mathrm{g} / \mathrm{ml}$ increase in plasma O3PUFA. These estimates indicate that an individual with an O3PUFA concentration of $200 \mu \mathrm{g} / \mathrm{ml}$ might be expected to complete the Trails B task $4 \mathrm{~s}$ faster than an individual with an O3PUFA of $100 \mu \mathrm{g} / \mathrm{ml}$ per year of aging. Given that each year of aging in our population was associated with a mean increase in Trails B completion time of approximately $4 \mathrm{~s}$ over the duration of follow up, these estimates indicate a 1 year delay in age-dependent executive decline per $100 \mu \mathrm{g} / \mathrm{ml}$ baseline O3PUFA concentration. By contrast, decline in Paragraph Recall ( $p=$ $0.83)$ and MMSE $(p=0.21)$ had no apparent relationship with O3PUFA.

Figure 1 illustrates the deceleration in trajectory of executive decline in people with "high" plasma O3PUFA versus people below this threshold. 
Table 2 | Plasma O3PUFA and cognitive decline in older adults over 4-years $(n=86)^{a}$.

\begin{tabular}{|c|c|c|c|c|c|c|c|c|c|}
\hline & \multicolumn{3}{|c|}{ Trail B } & \multicolumn{3}{|c|}{ Paragraph recall } & \multicolumn{3}{|c|}{ MMSE } \\
\hline O3PUFA baseline & -0.66 & 0.27 & 0.02 & 0.01 & 0.02 & 0.56 & 0.01 & 0.01 & 0.19 \\
\hline Age & 6.66 & 1.53 & 0.00 & -0.19 & 0.11 & 0.10 & -0.19 & 0.05 & 0.00 \\
\hline Gender & 14.61 & 10.81 & 0.18 & 1.56 & 0.83 & 0.06 & -0.05 & 0.22 & 0.81 \\
\hline Hypertension & 14.43 & 11.05 & 0.19 & -0.51 & 0.83 & 0.54 & -0.50 & 0.26 & 0.06 \\
\hline Depression & 11.44 & 14.28 & 0.42 & 0.95 & 1.09 & 0.38 & 0.05 & 0.34 & 0.88 \\
\hline
\end{tabular}

a Linear mixed effects model; age used as the time variable; O3PUFA at baseline includes plasma EPA+DHA; APOE4, apolipoprotein E epsilon 4 carrier; Gender, female; Education years; hypertension and depression require current treatment to qualify.

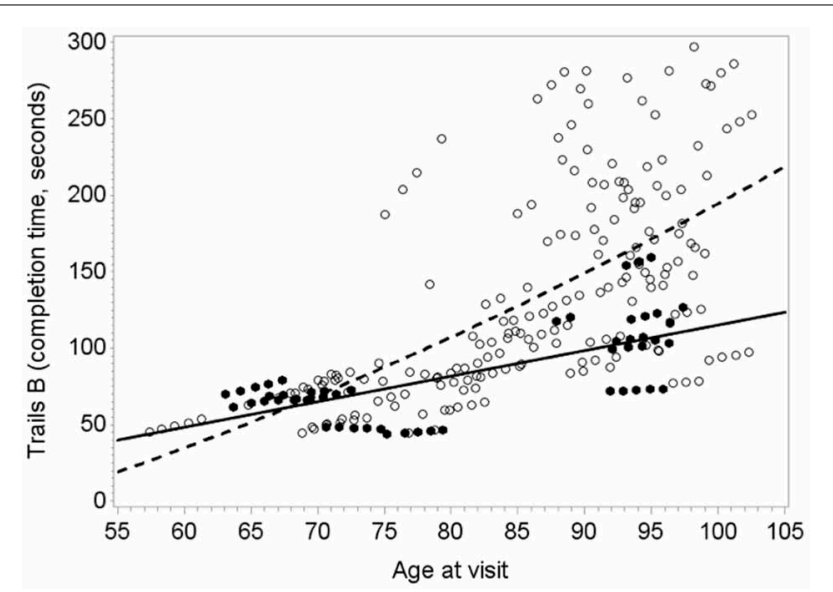

FIGURE 1 | Predicted within-person(circles) and mean trajectory (lines) of executive decline for subjects with plasma O3PUFA concentration above $110 \mu \mathrm{g} / \mathrm{ml}$ (dark circles, solidline) or below this threshold(open circles, dashed line) at base line $(n=86)$. Coefficient estimates

calculated in the linear mixed effects model were used to predict trajectories. Participants with O3PUFA $\leq 110 \mu \mathrm{g} / \mathrm{ml}$ (open circles and line) had an accelerated rate of executive decline by $2.7 \mathrm{~s}$ per year $(\beta=2.7 ; 95 \%$ confidence interval -5.11 to -0.22 ; age, gender, education, APOE4, hyper tension, depression adjusted).

\section{PLASMA 03PUFA AND WMH MEDIATED EXECUTIVE FUNCTION (FIGURE 2)}

O3PUFA associated with less WMH $(\beta=-0.188, p=0.007$, Figure 2A) where O3PUFA explained $28.5 \%$ of the variance in total WMH (Figure 3). WMH burden associated with worse executive function (Trials $B, \beta=4557.87, p=0.005$, Figure 2B). O3PUFA associated with better executive function (Tails $B$, $\beta=-1.15, p=0.025$, Figure $2 \mathrm{C}$ ), however, after adding $\mathrm{WMH}$ as a "mediating" variable into the regression equation that included O3PUFA, age, APOE4 and total intracranial volume simultaneously as predictors of executive function, the significant association between O3PUFA and better executive function was lost $(\beta=0.54, p=0.332$, Figure 2D) and the WMH association with executive dysfunction remained marginally significant ( $\beta=3589.95, p=0.056$, not illustrated in the Figure).

\section{DISCUSSION}

This longitudinal study of older adults at risk for dementia and followed over 4 years found significantly less executive decline in those with higher plasma O3PUFA at baseline. The calculated estimate indicates a 1-year delay in age-dependent executive decline per $100 \mu \mathrm{g} / \mathrm{ml}$ increase in plasma O3PUFA at baseline. We also found that plasma O3PUFA above $110 \mu \mathrm{g} / \mathrm{ml}$ associated with more stable executive function over time, which proposes one attainable threshold for neuroprotection. O3PUFA was not associated with verbal memory and MMSE. This implies that O3PUFA effects are more isolated to skills of executive function early on in people at risk for dementia. The mediation analysis further supports this notion since WMH accumulation is known to impact executive function and we demonstrate WMH mediation of the relationship between O3PUFA and executive dysfunction. Together, this evidence underlines important structural and functional brain parameters that seem well suited for targeting with O3PUFA therapy.

Delayed and logical verbal memory and MMSE changes were not associated with O3PUFA. However, executive decline appeared sensitive to O3PUFA. The mediation analysis is consistent with other literature indicating that prefrontal cortical executive skills are affected early during WMH accumulation (Schmidt et al., 1993; DeCarli et al., 1995; Adak et al., 2004; Brickman et al., 2006; Verdelho et al., 2007; Barbey et al., 2012). O3PUFA has pleiotropic effects that might explain this relationship, including effects on cerebral blood flow (Jackson et al., 2012), endothelial cell health (Yang et al., 2012), structural integrity of myelin (Pu et al., 2013), and preservation of neuronal energy with aging (Kuczynski et al., 2010).

Two other epidemiological studies have examined the relationship between O3PUFA and cognitive decline. Beydoun et al. (2007) were also unable to appreciate a relationship with global cognitive decline in the Atherosclerosis Risk in Communities Study. However, they did find O3PUFA associated with less decline in verbal/categorical fluency tasks which reflect executive control; requiring people to organize concepts in a novel way (i.e., naming words beginning with a particular letter or category with time constraints). They also report a more robust relationship in people with hypertension and dyslipidemia, two morbidities also 


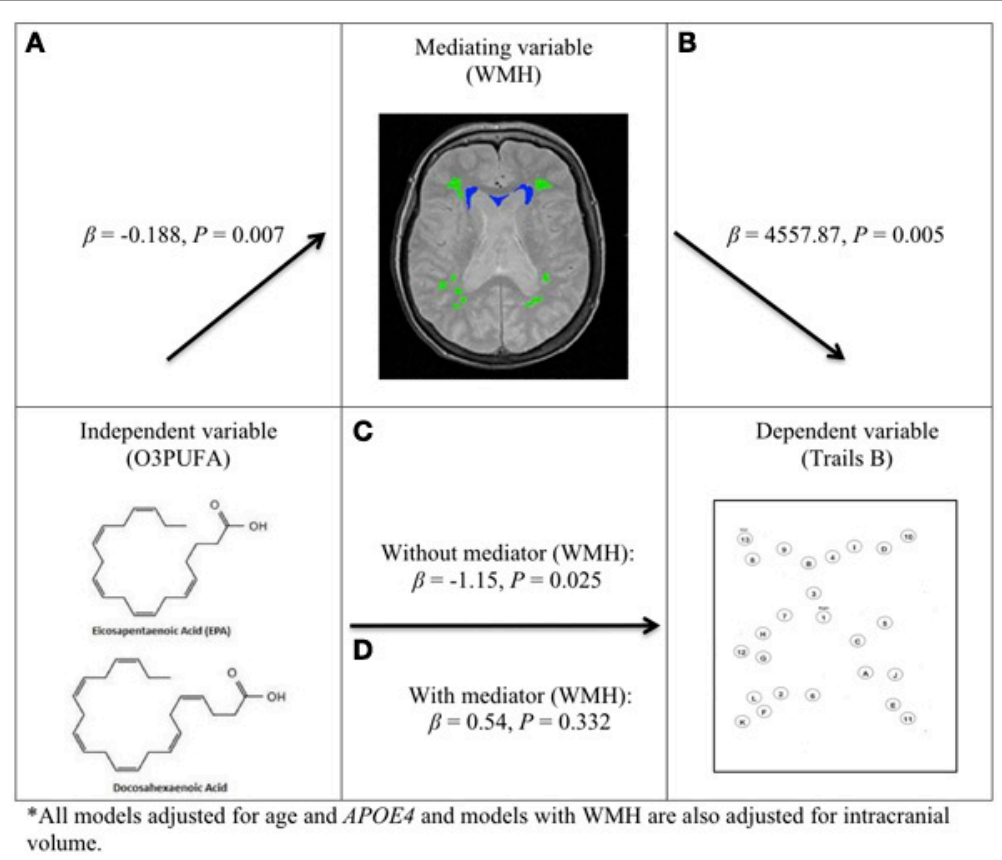

FIGURE 2 | O3PUFA and white matter mediated executive function in older adults $(\boldsymbol{n}=\mathbf{3 2})$. All models adjusted for age and APOE4, and models with WMH also adjusted for total intracranial volume. (A) Higher O3PUFA and less white matter hyper-intensities (WMH) highlighted in green (subcortical deep) and blue (periventricular). (B) Higher WMH and worse executive function (prolonged completion time for Trail B test). (C) Higher O3PUFA and better executive function (shorter completion time for Trail B test). (D) Association between O3PUFA and better executive function is lost once $\mathrm{WMH}$ is added to the regression model $(P=0.332$ ) representing mediation.

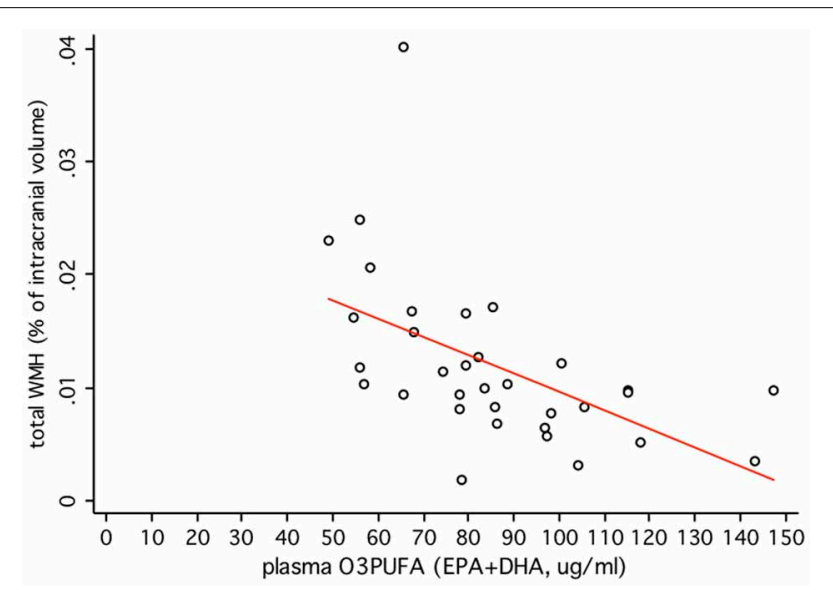

FIGURE 3 | Plasma O3PUFA explains $28.5 \%$ of the total variance in WMH in non-demented older adults $(n=32)$.

sensitive to O3PUFA (Mozaffarian and Wu, 2011). Samieri et al. (2011) did not observe a relationship between O3PUFA (DHA or EPA) and MMSE change over 7 years in a Bordeaux subset of the 3 City study or an association with executive decline represented by Trails B. This inconsistency with our results in OBAS may be attributed to several factors, including differences in O3PUFA measures themselves (EPA and DHA as a relative percentage of total fatty acids versus an absolute concentration that appears more informative in our OBAS sample that has both), the age of the cohort (mean age 74 vs. 86 in OBAS), slower annual rates of executive decline (about 1 s/year decline in Trails B vs. 4 s/year in OBAS), and the potential for lower $\mathrm{WMH}$ prevalence in the Bordeaux sample compared with OBAS.

Several clinical trials to prevent age-related cognitive decline in the cognitively intact using O3PUFA therapy have been completed (van de Rest et al., 2008; Dangour et al., 2010; Geleijnse et al., 2012) and others are underway (Danthiir et al., 2011). Each completed trial has been unsuccessful, however, the current study results suggest that the design of the clinical trials themselves may explain the null effect. For example, one trial enrolled non-demented subjects age 66-74 and followed subjects for only 6 months and executive function was examined (van de Rest et al., 2008). Another trial included secondary outcomes of executive function (e.g., digit span backwards and animal naming), but Trails B itself was not administered, and the population was at low vascular risk, younger age, and follow-up duration was limited at 2 years (Dangour et al., 2010). Geleijnse et al trial did examine a population with vascular risk (Geleijnse et al., 2012), but employed the MMSE as the primary outcome, which now has consistently shown to be insensitive to O3PUFA in observational and experimental studies of older adults at risk for dementia.

There were limitations in our study. We have plasma O3PUFA measurements available from a single time point in each participant, and we assume that this is a reasonable representation of long-term O3PUFA status, the type of nutritional exposure most inherently significant to brain aging. Our sample size is 
smaller than studies of cognitive aging and incident dementia using subjective measures of dietary intake (i.e., food frequency questionnaire). However, studies using self-report are susceptible to measurement error that we circumvent by utilizing quantitative nutrient biomarkers (Bowman et al., 2011; Tangney and Scarmeas, 2012). These permits more power, and, in turn a more conservative sample size to identify important relationships.

In conclusion, these results add longitudinal data to a limited body of literature that further indicate WMH and executive function as features of cognitive aging that appear sensitive to O3PUFA early in the evolution of cognitive decline. The hypothesis that O3PUFA can prevent vascular cognitive aging warrants further study.

\section{ACKNOWLEDGMENTS}

Study support provided by the NIH (K23-AT00004777, R01AG043398-01A1, RR024140) and the Portland VA Medical Center, Dr. Bowman conceptualized the study, led the acquisition and analysis of the fatty acids, drafted the analysis plan, interpreted the data, and composed the manuscript. His brain nutrition research is supported by the NIH (K23AT004777, R01AG043398-01A1) and Abbott Nutrition grants. Dr. Dodge supervised the data analysis and made substantive contribution in interpretation of results for the manuscript. She holds $\mathrm{NIH}$ support (K01 AG023014, P30 AG008017, R01 AG033581) and Scientific Review Board of the National Alzheimer's Coordinating Center duties. Dr. Silbert contributed in the interpretation of the brain scans and offered substantive contribution in the revision of the manuscript for intellectual content. She holds research support from the NIH (1R01AG036772, P30 AG008017, P50 NS062684) and receives Medicare and commercial insurance plan coverage for clinical care and intra-operative neurophysiological monitoring, and care provider at the Portland VA Medical Center. Dr. Barbey contributed in the interpretation of the neural mechanisms underlying prefrontal executive functions. Dr. Shinto receives salary and research support from the NIH, patient insurance reimbursement and made a substantive contribution in revising the manuscript for intellectual content. Ms. Nora Mattek reports no disclosures and made a substantive contribution in data quality assurance and technical details in the figures. Dr. Howieson assisted in the interpretation of neuropsychological evaluations and made a substantive contribution in revising the manuscript for intellectual content. She receives salary support from the NIH and insurance reimbursement from Medicare and other sources for providing patient care. Dr. Kaye assisted in the interpretation of the data and made a substantive contribution in revising the manuscript for intellectual content. He receives research support from the Department of Veterans Affairs (Merit Review grant) and the NIH (P30 AG008017, R01 AG024059, P30 AG024978, U01 AG010483); directs a center that receives research support from the NIH, Elan Corporation, Intel Corporation; receives reimbursement through Medicare and commercial insurance plans for providing patient care; is salaried to see patients at the Portland VA Medical Center; serves as an unpaid Chair for the Work Group on Technology and for the National Alzheimer's Association and as an unpaid Commissioner for the Center for Aging Services and Technologies; receives an annual royalty from sales of the book, Evidence-based Dementia Practice; and serves on the editorial advisory board of the journal, Alzheimer's \& Dementia. Dr. Quinn assisted in the interpretation of the data and made a substantive contribution in revising the manuscript for intellectual content. Dr. Quinn has received honoraria for speaking from Pfizer, Novartis, and Forrest and for consulting from Phylogeny, Inc. Dr. Quinn is a co-inventor on a patent for the use of DHA for the treatment of Alzheimer's disease. Dr. Quinn receives compensation for conducting clinical trials from Elan, Bristol Myers, and Baxter. Dr. Quinn receives funding from the $\mathrm{NIH}$ and Department of Veterans Affairs.

\section{SUPPLEMENTARY MATERIAL}

The Supplementary Material for this article can be found online at: http://www.frontiersin.org/journal/10.3389/fnagi. 2013.00092/abstract

\section{REFERENCES}

Adak, S., Illouz, K., Gorman, W., Tandon, R., Zimmerman, E. A., Guariglia, R. (2004). Predicting the rate of cognitive decline in aging and early Alzheimer disease. Neurology 63, 108-114. doi: 10.1212/01.WNL.0000132520. 69612.AB

Barbey, A. K., Colom, R., Solomon, J., Krueger, F., Forbes, C., and Grafman, J. (2012). An integrative architecture for general intelligence and executive function revealed by lesion mapping. Brain 135, 1154-1164. doi: 10.1093/brain/aws021

Baron, R. M., and Kenny, D. A. (1986). The moderator-mediator variable distinction in social psychological research: conceptual, strategic, and statistical considerations. J. Pers. Soc. Psychol. 51, 1173-1182. doi: 10.1037/00223514.51.6.1173

Bastos Leite, A. J., van der Flier, W. M., van Straaten, E. C., Scheltens, P., and Barkhof, F. (2006). Infratentorial abnormalities in vascular dementia. Stroke 37, 105-110. doi: 10.1161/01.STR.0000196984.90718.8a

Beydoun, M. A., Kaufman, J. S., Satia, J. A., Rosamond, W., and Folsom, A. R. (2007). Plasma n-3 fatty acids and the risk of cognitive decline in older adults: the Atherosclerosis Risk in Communities Study. Am. J. Clin. Nutr. 85, 1103-1111.

Bowman, G. L., Shannon, J., Ho, E., Traber, M. G., Frei, B., Oken, B. S., Kaye, J. A., and Quinn, J. F. (2011). Reliability and validity of food frequency questionnaire and nutrient biomarkers in elders with and without mild cognitive impairment. Alzheimer Dis. Assoc. Disord. 25, 49-57. doi: 10.1097/WAD.0b013e3181f333d6

Bowman, G. L., Silbert, L. C., Howieson, D., Dodge, H. H., Traber, M. G., Frei, B., et al. (2012). Nutrient biomarker patterns, cognitive function, and MRI measures of brain aging. Neurology 78, 241-249. doi: 10.1212/WNL.0b013e3182436598

Brickman, A. M., Zimmerman, M. E., Paul, R. H., Grieve, S. M., Tate, D. F., Cohen, R. A., et al. (2006). Regional white matter and neuropsychological functioning across the adult lifespan. Biol. Psychiatry 60, 444-453. doi: 10.1016/j.biopsych.2006.01.011

Dangour, A. D., Allen, E., Elbourne, D., Fasey, N., Fletcher, A. E., Hardy, P. et al. (2010). Effect of 2-y n-3 long-chain polyunsaturated fatty acid supplementation on cognitive function in older people: a randomized, double-blind, controlled trial. Am. J. Clin. Nutr. 91, 1725-1732. doi: 10.3945/ajcn.2009.29121

Danthiir, V., Burns, N. R., Nettelbeck, T., Wilson, C., and Wittert, G. (2011). The older people, omega-3, and cognitive health (EPOCH) trial design and methodology: a randomised, double-blind, controlled trial investigating the effect of long-chain omega-3 fatty acids on cognitive ageing and wellbeing in cognitively healthy older adults. Nutr. J. 10, 117. doi: 10.1186/1475-2891-10-117

Debette, S., Beiser, A., DeCarli, C., Au, R., Himali, J. J., Kelly-Hayes, M., et al. (2010). Association of MRI markers of vascular brain injury with incident stroke, mild cognitive impairment, dementia, and mortality: the Framingham Offspring Study. Stroke 41, 600-606. doi: 10.1161/STROKEAHA.109.570044

DeCarli, C., Murphy, D. G., Tranh, M., Grady, C. L., Haxby, J. V., Gillette, J. A., et al. (1995). The effect of white matter hyperintensity volume on brain structure, 
cognitive performance, and cerebral metabolism of glucose in 51 healthy adults. Neurology 45, 2077-2084. doi: 10.1212/WNL.45.11.2077

de Leeuw, F. E., de Groot, J. C., Achten, E., Oudkerk, M., Ramos, L. M., Heijboer, R., et al. (2001). Prevalence of cerebral white matter lesions in elderly people: a population based magnetic resonance imaging study. The Rotterdam Scan Study. J. Neurol. Neurosurg. Psychiatry 70, 9-14. doi: 10.1136/jnnp. 70.1 .9

Folstein, M. F., Folstein, S. E., and McHugh, P. R. (1975). “Mini-mental state”. A practical method for grading the cognitive state of patients for the clinician. J. Psychiatr. Res. 12, 189-198. doi: 10.1016/002-3956(75)90026-6

Garde, E., Lykke Mortensen, E., Rostrup, E., and Paulson, O. B. (2005). Decline in intelligence is associated with progression in white matter hyperintensity volume. J. Neurol. Neurosurg. Psychiatry 76, 1289-1291. doi: 10.1136/jnnp.2004.055905

Geleijnse, J. M., Giltay, E. J., and Kromhout, D. (2012). Effects of n-3 fatty acids on cognitive decline: A randomized, double-blind, placebo-controlled trial in stable myocardial infarction patients. Alzheimers Dement. 8, 278-287. doi: 10.1016/j.jalz.2011.06.002

Gorelick, P. B., Scuteri, A., Black, S. E., Decarli, C., Greenberg, S. M., Iadecola, C., et al. (2011). Vascular contributions to cognitive impairment and dementia: a statement for healthcare professionals from the american heart association/american stroke association. Stroke 42, 2672-2713. doi: 10.1161/STR.0b013 e3182299496

Jackson, P. A., Reay, J. L., Scholey, A. B., and Kennedy, D. O. (2012). Docosahexaenoic acid-rich fish oil modulates the cerebral hemodynamic response to cognitive tasks in healthy young adults. Biol. Psychol. 89, 183-190. doi: 10.1016/j.biopsycho.2011.10.006

Kaye, J. A., Oken, B. S., Howieson, D. B., Howieson, J., Holm, L. A., and Dennison, K. (1994). Neurologic evaluation of the optimally healthy oldest old. Arch. Neurol. 51, 1205-1211. doi: 10.1001/archneur.1994.00540240049015

Kiernan, R. J., Mueller, J., Langston, J. W., and Van Dyke, C. (1987). The Neurobehavioral Cognitive Status Examination: a brief but quantitative approach to cognitive assessment. Ann. Intern. Med. 107, 481-485. doi: 10.7326/0003-4819-107-4-481

Kramer, J. H., Mungas, D., Reed, B. R., Wetzel, M. E., Burnett, M. M., Miller, B. L., Weiner, M. W., and Chui, H. C. (2007). Longitudinal MRI and cognitive change in healthy elderly. Neuropsychology 21, 412-418. doi: 10.1037/08944105.21.4.412

Kuczynski, B., Targan, E., Madison, C., Weiner, M., Zhang, Y., Reed, B., et al. (2010). White matter integrity and cortical metabolic associations in aging and dementia. Alzheimers Dement. 6, 54-62. doi: 10.1016/j.jalz.2009.04.1228

Morris, J. C. (1993). The Clinical Dementia Rating (CDR): current version and scoring rules. Neurology 43, 2412-2414. doi: 10.1212/WNL.43.11.2412-a

Mozaffarian, D., and Wu, J. H. (2011). Omega-3 fatty acids and cardiovascular disease: effects on risk factors, molecular pathways, and clinical events. J. Am. Coll. Cardiol. 58, 2047-2067. doi: 10.1016/j.jacc.2011.06.063

Mueller, E. A., Moore, M. M., Kerr, D. C., Sexton, G., Camicioli, R. M., Howieson, D. B., et al. (1998). Brain volume preserved in healthy elderly through the eleventh decade. Neurology 51, 1555-1562. doi: 10.121/WNL. 51.6.1555

Pu, H., Guo, Y., Zhang, W., Huang, L., Wang, G., Liou, A. K., et al. (2013). Omega3 polyunsaturated fatty acid supplementation improves neurologic recovery and attenuates white matter injury after experimental traumatic brain injury. J. Cereb. Blood Flow Metab. 33, 1474-1484. doi: 10.1038/jcbfm.2013.108

Reitan, R. M. (1958). Validity of the trail making test as an indicator of organic brain damage. Percept. Motor Skills 8, 271-276.

Samieri, C., Feart, C., Proust-Lima, C., Peuchant, E., Dartigues, J. F., Amieva, H., et al. (2011). Omega-3 fatty acids and cognitive decline: modulation by ApoEepsilon4 allele and depression. Neurobiol. Aging 32, 2317 e13-2317 e22. doi: 10.1016/j.neurobiolaging.2010.03.020

Schmidt, R., Fazekas, F., Offenbacher, H., Dusek, T., Zach, E., Reinhart, B., et al. (1993). Neuropsychologic correlates of MRI white matter hyperintensities: a study of 150 normal volunteers. Neurology 43, 2490-2494. doi: 10.1212/WNL.43.12.2490
Schmidt, R., Schmidt, H., Kapeller, P., and Fazekas, F. (2003). Slow progression of white-matter changes. Int. Psychogeriatr. 15(Suppl. 1), 173-176. doi: 10.1017/S1041610203009153

Silbert, L. C., Dodge, H. H., Perkins, L. G., Sherbakov, L., Lahna, D., ErtenLyons, D., et al. (2012). Trajectory of white matter hyperintensity burden preceding mild cognitive impairment. Neurology 79, 741-747. doi: 10.1212/WNL.0b013e3182661f2b

Silbert, L. C., Howieson, D. B., Dodge, H., and Kaye, J. A. (2009). Cognitive impairment risk: white matter hyperintensity progression matters. Neurology 73, 120-125. doi: 10.1212/WNL.0b013e3181ad53fd

Silbert, L. C.,Nelson, C., Howieson, D. B., Moore, M. M., and Kaye, J. A. (2008). Impact of white matter hyperintensity volume progression on rate of cognitive and motor decline. Neurology 71, 108-113. doi: 10.1212/01.wnl.0000316799.86917.37

Tan, Z. S., Harris, W. S., Beiser, A. S., Au, R., Himali, J. J., Debette, S., et al. (2012). Red blood cell omega-3 fatty acid levels and markers of accelerated brain aging. Neurology 78, 658-664. doi: 10.1212/WNL.0b013e318 $249 \mathrm{f} 6 \mathrm{a} 9$

Tangney, C. C., and Scarmeas, N. (2012). The good, bad, and ugly?: How blood nutrient concentrations may reflect cognitive performance. Neurology 78, 230-231. doi: 10.1212/WNL.0b013e31824367da

van de Rest, O., Geleijnse, J. M., Kok, F. J., van Staveren, W. A., Dullemeijer, C., Olderikkert, M. G., et al. (2008). Effect of fish oil on cognitive performance in older subjects: a randomized, controlled trial. Neurology 71, 430-438. doi: 10.1212/01.wnl.0000324268.45138.86

Verdelho, A., Madureira, S., Ferro, J. M., Basile, A. M., Chabriat, H., Erkinjuntti, T., et al. (2007). Differential impact of cerebral white matter changes, diabetes, hypertension and stroke on cognitive performance among non-disabled elderly. The LADIS study. J. Neurol. Neurosurg. Psychiatry 78, 1325-1330. doi: 10.1136/jnnp.2006.110361

Virtanen, J. K., Siscovick, D. S., Lemaitre, R. N., Longstreth, W. T., Spiegelman, D., Rimm, E. B., et al. (2013). Circulating omega-3 polyunsaturated fatty acids and subclinical brain abnormalities on MRI in older adults: the cardiovascular health study. J. Am. Heart Assoc. 2, e000305. doi: 10.1161/JAHA.113. 000305

Virtanen, J. K., Siscovick, D. S., Longstreth, W. T., Kuller, L. H. Jr., and Mozaffarian, D. (2008). Fish consumption and risk of subclinical brain abnormalities on MRI in older adults. Neurology 71, 439-446. doi: 10.1212/01.wnl.0000324414.12665.b0

Wechsler, D. (1981). Manual for the Wechsler Adult Intelligence Revised. San Antonio: The Psychological Corporation.

Yang, Y., Lu, N., Chen, D., Meng, L., Zheng, Y., and Hui, R. (2012). Effects of n-3 PUFA supplementation on plasma soluble adhesion molecules: a metaanalysis of randomized controlled trials. Am. J. Clin. Nutr. 95, 972-980. doi: 10.3945/ajcn.111.025924

Conflict of Interest Statement: The authors declare that the research was conducted in the absence of any commercial or financial relationships that could be construed as a potential conflict of interest.

Received: 22 September 2013; accepted: 27 November 2013; published online: 16 December 2013.

Citation: Bowman GL, Dodge HH, Mattek N, Barbey AK, Silbert LC, Shinto L, Howieson DB, Kaye JA and Quinn JF (2013) Plasma omega-3 PUFA and white matter mediated executive decline in older adults. Front. Aging Neurosci. 5:92. doi: 10.3389/ fnagi.2013.00092

This article was submitted to the journal Frontiers in Aging Neuroscience. Copyright (C) 2013 Bowman, Dodge, Mattek, Barbey, Silbert, Shinto, Howieson, Kaye and Quinn. This is an open-access article distributed under the terms of the Creative Commons Attribution License (CC BY). The use, distribution or reproduction in other forums is permitted, provided the original author(s) or licensor are credited and that the original publication in this journal is cited, in accordance with accepted academic practice. No use, distribution or reproduction is permitted which does not comply with these terms. 